\title{
Structural Identification using a Nonlinear Constraint Satisfaction Processor with Interval Arithmetic and Contractor Programming
}

\author{
Timothy Kernicky ${ }^{\mathrm{a}}$, Matthew Whelan ${ }^{\mathrm{a}}$, Usman Rauf ${ }^{\mathrm{b}}$, Ehab Al-Shaer ${ }^{\mathrm{b}}$ \\ ${ }^{a}$ University of North Carolina at Charlotte, Department of Civil and Environmental Engineering, \\ 9201 University City Boulevard, Charlotte, NC 28223-0001 U.S.A. \\ ${ }^{b}$ University of North Carolina at Charlotte, Department of Software and Information Systems, \\ 9201 University City Boulevard, Charlotte, NC 28223-0001 U.S.A.
}

\begin{abstract}
Structural identification through finite element model updating has gained increased importance as an applied experimental technique for performance-based structural assessment and health monitoring. However, practical challenges associated with computability, feasibility, and uniqueness present in the structured nonlinear inverse eigenvalue problem develop as a result of the necessary use of partially described and incompletely measured mode shapes. As an alternative to direct methods and optimization-based approaches, this paper proposes a new paradigm for model updating that is based on formulating the structured inverse eigenvalue problem as a Constraint Satisfaction Problem. Interval arithmetic and contractor programming are introduced as a means for generating feasible solutions to a structured inverse eigenvalue problem within a bounded parameter search space. This framework offers the ability to solve under-determined and non-unique inverse problems as well as accommodate measurement uncertainty through relaxation of constraint equations. These abilities address key challenges in quantifying uncertainty in parameter estimates obtained through structural identification and enable the exploration of alternative solutions to the global minimum that may better reflect the true physical properties of the structure. These capabilities are first demonstrated using synthetic data from a numerical mass-spring model and then extended to experimental data from a laboratory shear building model. Lastly, the methodology is contrasted with probabilistic model updating to highlight the advantages and unique capabilities offered by the methodology in addressing the effects of measurement uncertainty on the parameter estimation.
\end{abstract}

Keywords: Structural Identification, Finite Element Model Updating, Partially Described Inverse Eigenvalue Problem, Interval Arithmetic, Vibration-Based Structural Health Monitoring

\section{Introduction}

Over the past several decades, numerous techniques have been devised to develop updated structural stiffness and mass matrices from modal parameter estimates obtained from either experimental or operational modal analysis. Within structural identification, the properties of the updated model are used to infer the behavior and performance of the structure to inform decision-making [1]. Consequently, instilling confidence that parameter assignments in the updated model closely reflect physical reality is critical to the use of structural identification for applications in performance-based civil engineering and structural health monitoring. Likewise, understanding the uncertainty in the parameter estimates in the presence of measurement noise and potential ill-conditioning of the inverse problem is necessary to provide reliable and actionable information.

Traditionally, the finite element model updating problem has been framed using the generalized eigenvalue problem for undamped multiple degree of freedom linear systems:

$$
K \Phi=M \Phi \Omega^{2}
$$

where $M$ and $K$ are the mass and stiffness matrices, $\Omega^{2}$ is a diagonal matrix containing the eigenvalues $\left(\omega_{n}^{2}\right.$, where $\omega_{n}$ are the undamped natural frequencies) on the diagonal, and $\Phi$ is the matrix containing the corresponding eigenvectors, 
or mode shapes, as columns of the matrix. This generalized eigenvalue problem has been adopted for the majority of structural identification applications since the finite element method can be used to readily construct the mass and stiffness matrices, while experimentally measured relative damping factors can be used to construct the corresponding damping matrix for the system. The general objective of model updating is to modify the stiffness and mass matrices of an analytical model of the structure such that the eigenproperties of the model obtain the best possible match to experimentally measured values, while preserving physical meaning and structural constraints in the matrices. Although this objective is easily described, there are many practical challenges that arise from the nature of vibration testing and analytical modeling that are ubiquitous to all methods employed for model updating. Namely:

- The practical measurement bandwidth of vibration transducers is limited, which effectively limits the measurement of the eigenvalues and eigenvectors of the system under test to typically a small subset of all of those that would exist in the corresponding analytical model. This case is commonly referred to as having partially described eigeninformation pairs [2].

- It is impractical to completely measure all corresponding degrees of freedom in the analytical model, so the experimental mode shapes are incomplete measurements of the eigenvector. For models of even modest size structures, the number of sensors required to measure every degree of freedom in a sufficiently discretized finite element model is generally prohibitive. Furthermore, the direct measurement of some rows of the eigenvector, such as those associated with the rotational degrees of freedom, is not even possible with conventional transducers [3].

- Noise and uncertainty in the measurements, as well as assumptions inherent to system identification algorithms, yield mode shapes that no longer satisfy orthogonality relationships and are unlikely to satisfy equality constraints. Likewise, discretization errors and idealizations inherent to the model, such as element type and mesh connectivity, are not explicitly corrected in conventional finite element model updating schemes [4].

Currently, the two most prevalent techniques applied for structural identification are deterministic and probabilistic methods of finite element model updating. Deterministic methods seek to identify optimal assignments for a set of uncertain parameters in the model by minimizing the residuals between measured and estimated modal parameters by application of various optimization techniques $[5,6,7]$. While these techniques have been applied for structural identification of several full-scale structures $[8,9]$, their application is generally plagued by issues associated with computational speed, solution uniqueness, ill-conditioning, and parameter selection and sensitivity [10]. Furthermore, finite element model updating problems suffer from the underlying issue that the global minimum may not necessarily reflect the best match to the physical reality due to uncorrected errors arising from idealization and discretization in the model and uncertainties in the measurements [4]. These challenges have given rise to probabilistic finite element model updating approaches [11], which incorporate uncertainties in the model and the measurements to identify the most probable solutions using statistical methods. However, these probabilistic methods require an assumed probability density function for uncertain variables and often require computationally expensive simulations to arrive at the solution [7]. Lastly, it should be noted that direct methods for solving inverse eigenvalue problems with incompletely measured mode shapes through solution of a descriptor Sylvester equation have recently emerged $[12,13]$, however their application has yet to demonstrate the capability to preserve the connectivity structure of the matrices.

Although probabilistic approaches have been the most prevalently used to address uncertainties in structural models, interval methods provide an alternative approach that may offer computational advantages over probabilistic techniques $[14,15]$. Recently, interval-based model updating strategies have been proposed for handling inherent uncertainties in the experimental modal parameter estimates and the finite element model [16]. The most closely related work to the current study leverages interval global optimization to arrive at solutions to the model updating problem [17]. However, the technique proposed in this prior work is formulated on an inclusion set for the eigenvalues of an interval stiffness matrix and employs a simple branch and bound algorithm to minimize an objective function rather than solve a constraint satisfaction problem. An extension of this work incorporated interval eigenvectors in the optimization through an acceptance criteria based on the modal assurance criterion [18]. It should also be noted that the term "interval model updating" has also been recently used to described the application of interval arithmetic techniques in the estimation of parameter variability $[19,20]$. However, these approaches address the field of stochastic model updating [21], wherein the objective is to characterize the variabilities across a number of experimental tests 
performed on nominally identical structures. While the constraint satisfaction formulation proposed in the current work may have extensions to stochastic model updating, the subject of this current paper is on the class of model updating applications where measurements obtained from a single structure are used to calibrate a numerical model.

This paper presents a novel formulation of the finite element model updating problem as a constraint satisfaction problem and explores the use of a nonlinear constraint satisfaction processor with interval arithmetic and contractor programming to yield estimates of uncertain model parameters and unmeasured components of the eigenvectors. The method is shown to be capable of delivering a complete set of feasible solutions to the structured inverse eigenvalue problem with partially described and incompletely measured eigeninformation pairs from either synthetic or experimental data. Furthermore, the approach is successfully demonstrated on ill-posed problems with multiple solutions to illustrate its capability for addressing this challenge as well as providing a foundation to introduce practitioner heuristics into the identification of physically plausible solution sets.

\section{Nonlinear Constraint Satisfaction with Contractor Programming and Interval Analysis}

In the domain of engineering sciences, many applications require finding all possible and potentially isolated solutions satisfiable to a set of constraints over real numbers. The system of equations may be non-polynomial and the computational complexity to solve such systems is NP-hard. This set of problems are called Constraint Satisfaction Problems (CSPs) [22, 23, 24]. The approach explored in this paper for the solution of partially described and incompletely measured inverse eigenvalue problems relies on framing the structured inverse eigenvalue problem as a nonlinear CSP. By developing the model updating problem in this framework, unique capabilities for addressing challenges related to ill-posedness and ill-conditioning are revealed, as detailed in later sections. The following discussion provides some details on the fundamentals of CSPs, interval analysis, and contractor programming that are essential for the understanding of the rest of the paper.

Fundamentally, a CSP can be defined as a 4-tuple $<V, D, C, L>$ where:

- $V=\left\{v_{1}, v_{2}, \ldots, v_{n}\right\}$ is a set of variables

- $D=\left\{d_{1}, d_{2}, \ldots, d_{n}\right\}$ is a set of domains for prospective variables

- $C=\left\{c_{1}, c_{2}, \ldots, c_{n}\right\}$ is a set of constraints over the variables

- $L$ is a set of labels that map constraints to the variables and corresponding domains, formally: $L: C_{i} \rightarrow\left(v_{i}, d_{i}\right)$

Each variable, $v_{i}$, can assume any real value in the corresponding non-empty domain $d_{i}$. The constraint $c_{i} \in C$ is defined over a pair $\left(v_{i}, d_{i}\right)$ through a label function $l \subset L$. In the process of finding a satisfiable solution to a CSP, different values are assigned to the variables (in set $V$ ) from the domain set $(D)$ through constraint propagation algorithms (also called filtering/contraction algorithms). The evaluation of label (true/false) determines whether the corresponding assignments to variables for a given constraint lead to a satisfiable solution or not. While assigning values to different variables (through a "systematic search process"), consistency algorithms [25] take into account whether any violation of constraints has occurred during any assignment. This whole process is called constraint propagation. Likewise, the paradigm for solving CSPs through integration of Systematic Search and Arc Consistency techniques is called Constraint Programming (CP).

A sub-domain of constraint programming that integrates interval arithmetic, called Interval Constraint Programming, offers unique advantages for developing solutions to the inverse eigenvalue problem with experimental data. In interval arithmetic, variables as well as constraint relationships among variables can be defined using intervals and mathematical operations can be applied using principles of interval arithmetic [26]. Consequently, interval-based computation offers a means of placing bounds on round off errors in numerical calculations, measurement uncertainties, and uncertain model parameters. Therefore, rather than defining the exact value of a variable or constraint, it can be defined over an interval, which implies that it may take any value within that interval. The Cartesian product of intervals, or interval vector, generates a multi-dimensional volume, or box. Formally, a variable " $\mathrm{X}$ " defined over an interval and the realization of interval vectors can be explained as follows:

$$
\begin{aligned}
X:[a, b] & \rightarrow\{X \in \mathfrak{R} \mid a \leq X \leq b\} \\
{[X] } & =\left[x_{1}\right] \times\left[x_{2}\right] \ldots \times\left[x_{n}\right]
\end{aligned}
$$

and is illustrated graphically in Figure 1a for the product of two intervals. In an interval constraint programming problem, the objective is to completely and correctly identify all interval vectors, to a specified interval precision, that satisfy the set of constraint equations within the bounded domains for each set of interval variables. 


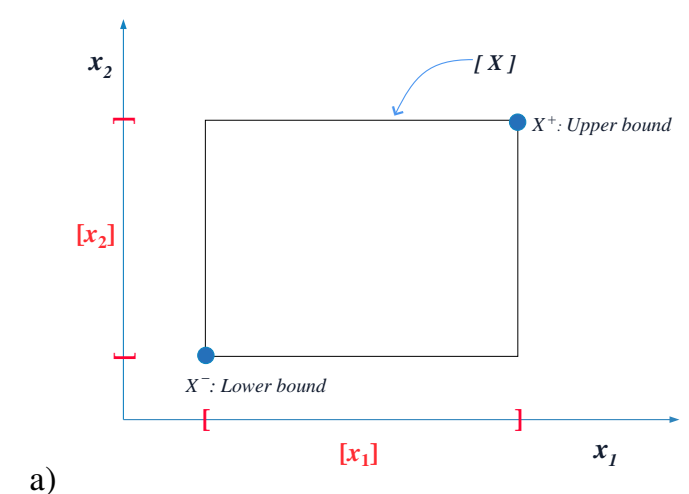

a)

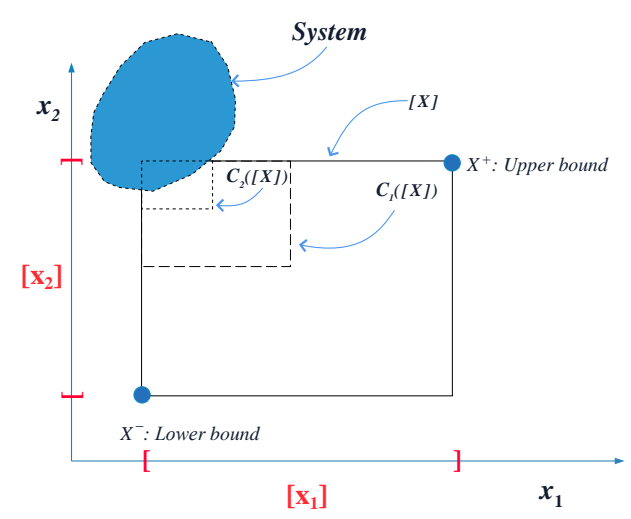

Figure 1: a) An interval vector, or box, generated from the product of two intervals. b) contraction of an interval vector on a system of constraints

\subsection{Contractor Programming}

One recent effort toward the integration of interval arithmetic and constraint programming in a formalized solver has been termed Contractor Programming [27]. In this approach, contractors can be viewed as mathematical operators that accept a system (an n-dimensional box) as an input and contract the domain of the system according to a given set of constraints. Contractors yield a reliable set of satisfiable solutions since they must satisfy properties of soundness and completeness. The formal definition of a contractor and its properties are defined as follows:

A contractor $(C)$ is a function from $C: I \mathfrak{R}^{n} \rightarrow I \mathfrak{R}^{n}$, where $I \mathfrak{R}$ is interval over reals, with the following properties:

- $\forall[X] \in I \Re^{n} \quad C([X]) \subset[X]$; where $C([X])$ represents box $[X]$ after contraction

- $C([X]) \cap$ System $=[X] \cap$ System

The first property ensures that the contraction algorithm or procedure is sound and that the result achieved after contraction contains a subset of the original box (Figure 1b). The second property elaborates the completeness of the contraction algorithm/procedure, which states that no potential solution is lost after contraction is performed. Adhering to these two properties, contractor programming yields reliable results with a guarantee that feasible solutions are not lost within the search space. Following contraction, the interval vector can be bisected into a subset of smaller boxes where contractors can be applied to further refine the interval precision of the solution. In this respect, solvers employing contractor programming have some parallels to branch and bound algorithms, where subsets of a search space are evaluated and either retained or discarded as potential solutions and then the retained subsets are further divided and evaluated until the desired interval precision is obtained. However, the process of contraction can result in a significantly faster exploration of the search space than pure branching methods, such as bisection [28].

Although many domain experts in structural engineering will be unfamiliar with the concept of constraint programming and contractors, the general application to a variety of challenging nonlinear engineering problems has been made possible through the open-source IBEX $\mathrm{C}++$ numerical library [29]. For the current study, the authors have developed an interface between the MATLAB interactive environment [30] and the IBEX 2.1.17 library to construct and parameterize the structural stiffness and mass matrices, develop mechanics-based constraint equations, pass the formulated CSP to the IBEX solver, and parse any and all interval solutions generated by the contraction algorithm for subsequent analysis. In this study, the generic default solver in this release of IBEX, which employs forward-backward contraction through the HC4 algorithm [31] and adaptive constructive interval disjunction [32], has been used for all of the examples presented to promote accessibility of the developed model updating formulation to a broader audience. Details on the implementation for finite element model updating are presented following a short illustrative example demonstrating the key benefits sought from this alternative to optimization-based strategies for inverse problems. 


\subsection{Illustrative Example Using Cross-in-Tray Function}

A brief example illustrating the computational advantages offered by contractor programming techniques is presented to provide motivation for its use in finite element model updating. Specifically, the finite element model updating challenges related to ill-posedness and the desire to obtain alternative solutions in the presence of experimental noise and uncertainty are highlighted by use of a nonlinear function with a large number of local extrema, multiple global minima, and discontinuities. For this illustration, we consider the Cross-in-Tray function:

$$
f(x, y)=-0.0001\left(\left|\sin (x) \sin (y) \exp \left(\left|100-\frac{\sqrt{x^{2}+y^{2}}}{\pi}\right|\right)\right|+1\right)^{0.1}
$$

which has been adopted as a benchmark for performance testing of optimization algorithms and features four global minima at $\{x, y\}=( \pm 1.34941, \pm 1.34941)$ corresponding to a function value of -2.06261 . The domain of the search space was bounded to $x \in[-10,10]$ and $y \in[-10,10]$, which corresponds to the region shown in Figure 2a. This function was first encoded in an equality constraint equation where satisfiable solutions were required to adhere to the exact function minimum and the IBEX constraint processor successfully identified all four global minima in approximately 0.02 seconds. This ability to identify multiple solutions by contractor programming will be leveraged to overcome uniqueness challenges in the inverse eigenvalue problem. In addition, by relaxing the equality constraint to an inequality constraint, $f(x, y) \leq-1.75$, the solver demonstrates the ability to identify all feasible alternative solutions to the unknown parameters $x$ and $y$ that yield results similar to the global minima of this challenging nonlinear and discontinuous function within half of a second (Figure 2b). This ability to completely identify all feasible solutions to a set of relaxed constraint equations will be leveraged to address challenges associated with measurement uncertainties and the effects of potential ill-conditioning in the finite element model updating problem. Note that the boxes shown in this plot reflect the bisection and contraction processes performed to arrive at the interval solutions, which illustrates the computational efficiency over pure branch and bound algorithms.
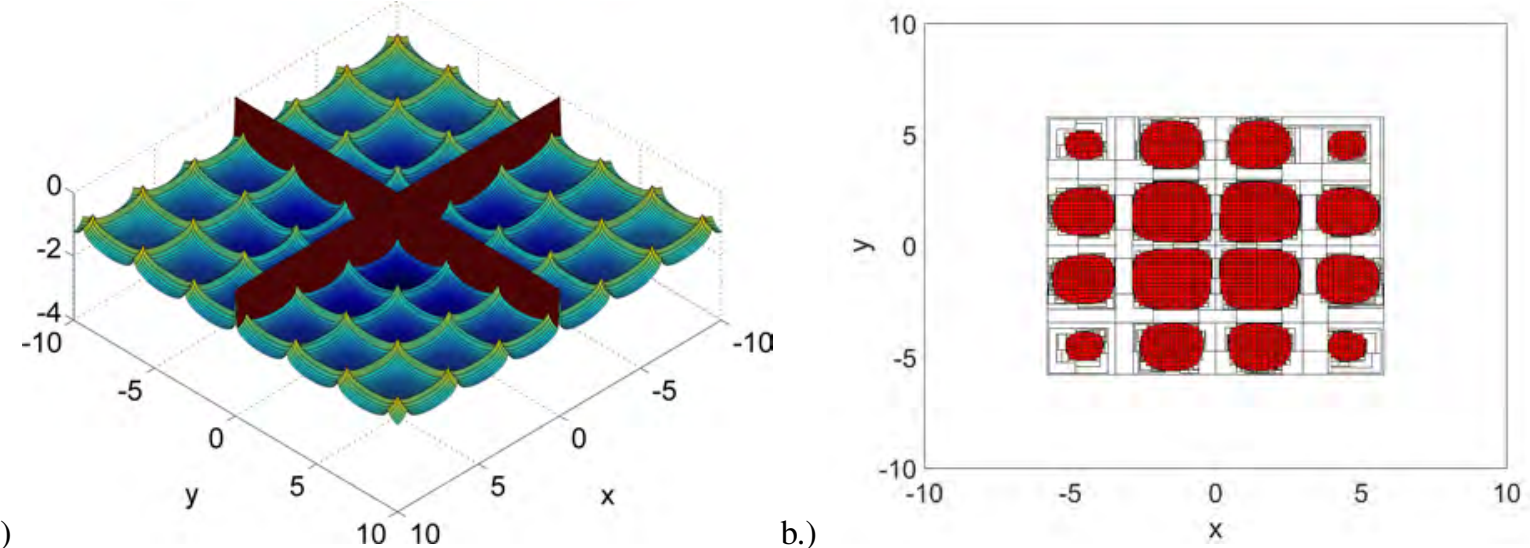

Figure 2: a.) Cross-in-Tray Function, b.) Set of satisfiable solutions produced with relaxed inequality constraint using contractor programming (all boxes shown represent interval vectors after subsequent contractions and shaded boxes indicate satisfiable solutions)

\section{Formulation of Interval Constraint Equations for the Structured Inverse Eigenvalue Problem}

The real-valued inverse eigenvalue problem aims to solve for the symmetric matrices $M$ and $K$, which are positivedefinite and positive-semidefinite, respectively, given specified eigeninformation matrices $\left(\Omega^{2}, \Phi\right)$. Within the application to finite element model updating, additional conditions are generally applied to the inverse eigenvalue problem, such as conditions to maintain the sparsity structure of the matrices to ensure proper connectivity of elements in the model and to ensure physically meaningful solutions. In our proposed approach to model updating, we formulate the generalized eigenvalue problem as a matrix-based constraint equation and apply additional mechanics-based constraint equations to enforce desired qualities on the structural matrices associated with the updated model. In the 
generalized eigenvalue problem for undamped multiple degree-of-freedom (MDOF) discrete systems, the structural matrices are $n \times n$ square, where $n$ is the number of unrestrained degrees of freedom in the model. Numerically, eigendecomposition of these matrices will produce $n$ eigenpairs. However, in practical applications of model updating it is generally prohibitive to obtain corresponding measurements of all of these eigenpairs due to a variety of experimental limitations, including the measurement bandwidth of conventional vibration transducers, insufficient excitation of all modes, and restrictions on the number of sensors available. Therefore, the majority of model updating problems are classified as partially described, where only $m<n$ eigenpairs are measured. Under these conditions, the constraint equations developed for the inverse eigenvalue problem must necessarily be formulated using only $m$ modes, as:

$$
K_{n \times n} \Phi_{n \times m}-M_{n \times n} \Phi_{n \times m} \Omega_{m \times m}^{2}=0_{n \times m}
$$

Consequently, the partially described eigenvalue problem yields a total of $n \times m$ unique constraint equations. However, since it is generally impractical or impossible to measure all $n$ degrees of freedom associated with the analytical model being updated, the eigenvectors included in these constraint equations will be incompletely measured. As a result, the number of unknowns in the eigenvector matrix will be of size $(n-s) \times m$, where $s$ corresponds to the number of measured degrees of freedom or sensors, and the matrix constraint equation can be partitioned as:

$$
K_{n \times n}\left[\begin{array}{c}
\Phi_{s \times m}^{M} \\
\Phi_{(n-s) \times m}^{U}
\end{array}\right]-M_{n \times n}\left[\begin{array}{c}
\Phi_{s \times m}^{M} \\
\Phi_{(n-s) \times m}^{U}
\end{array}\right] \Omega_{m \times m}^{2}=0_{n \times m}
$$

where $\Phi_{s \times m}^{M}$ are the rows of the measured components of the mode shapes and $\Phi_{(n-s) \times m}^{U}$ are the rows of the unmeasured components of the mode shapes. Conventional approaches for linear parameterization of the structural matrices can also be introduced to the governing constraint equations to enforce additional mechanics-based constraints on the updated model. In the formulation of generalized eigenvalue problem employed for all numerical and experimental examples in this paper, the structural matrices are explicitly formed through elemental contributions, or basis matrices. Consequently, the constraints on the sparsity pattern of the structural matrices are explicitly encoded in Eq. 5 to produce:

$$
\left[K_{o}+\sum_{j=1}^{N_{K}} \alpha_{j} K_{j}\right]\left[\begin{array}{c}
\Phi_{s \times m}^{M} \\
\Phi_{(n-s) \times m}^{U}
\end{array}\right]-M_{n \times n}\left[\begin{array}{c}
\Phi_{s \times m}^{M} \\
\Phi_{(n-s) \times m}^{U}
\end{array}\right] \Omega_{m \times m}^{2}=0_{n \times m}
$$

where $\alpha_{j}$ is a scalar multiplier applied to the basis matrix, $K_{j}$, that provides the stiffness contributions of the $j$-th element of the model, while $K_{o}$ represent the contributions of any elements in the model not subject to updating. In this formulation, the unknowns in the inverse eigenvalue problem are the $N_{K}$ scalar multipliers and the $(n-s) \times m$ unmeasured components of the eigenvector matrix. Using interval arithmetic and contractor programing, the solutions for these unknown quantities that satisfy the constraint equation in Equation 6 within a bounded search space will be determined to a prescribed interval precision, or width. Consequently, the methodology develops an interval stiffness matrix, $K^{I}$, since the uncertain parameters in the model are treated as the intervals:

$$
\alpha=[\underline{\alpha}, \bar{\alpha}]
$$

where $\underline{\alpha}$ is the lower bound to the interval for the uncertain parameter and $\bar{\alpha}$ is the upper bound. Likewise, the unknown components of the eigenvector matrix are treated as intervals that are subsequently bisected and contracted until the desired precision of the solution is achieved:

$$
\Phi_{(n-s) \times m}^{U}=\left[\underline{\Phi}_{(n-s) \times m}^{U}, \bar{\Phi}_{(n-s) \times m}^{U}\right]
$$

It should be noted that, in addition to the stiffness matrix, the mass matrix can also be parameterized. The authors have successfully implemented such cases, however the current paper focuses only on parameterization of the stiffness matrix to yield results with a physical basis that is more readily interpreted for cases with multiple solutions.

In the presence of measurement uncertainty and idealization errors in the model, an additional set of constraint equations must also be introduced to ensure that the prescribed eigenvalues are consistent with the stiffness and mass matrices assembled with the interval uncertain parameter assignments. In other words, while feasible solutions to the equality constraints in Equation 6 with noise-free eigeninformation pairs guarantee that the prescribed values in $\Omega_{n \times m}^{2}$ 
are contained within the interval eigenvalues of the mass and stiffness matrix, this is not guaranteed if $\Phi_{s \times m}^{M}$ are not exact eigenvectors of the structural matrices. Consequently, an additional set of constraints, which we refer to herein as the eigenvalue inclusion constraints, must be introduced to ensure that the specified eigenvalues are contained within the interval eigenvalues of the structural matrices. However, solving for the eigenvalues of systems described by interval mass and stiffness matrices requires iterative methods to perform the eigenvalue decomposition that are not compatible with the direct arithmetic operations used within contractor programming to contract the interval vectors. In order to overcome this limitation, the specified eigenvalues are checked for inclusion by substituting each into the characteristic equation formed from the determinate:

$$
\left|K^{I}-\omega_{r}^{2} M\right|=\left|K_{o}+\sum_{j=1}^{N_{K}}\left[\underline{\alpha_{j}}, \overline{\alpha_{j}}\right] K_{j}-\omega_{r}^{2} M\right|=0
$$

Finally, in addition to these eigenvalue inclusion constraints, orthogonality constraints are introduced to aid with the identification of the unmeasured components of each mode shape. For each of the eigenvectors included in the prescribed eigeninformation matrix, $\phi$, the following constraint equations can be enforced:

$$
\begin{array}{r}
\phi_{r}^{T} M \phi_{q}=0, r \neq q \\
\phi_{r}^{T} K^{I} \phi_{q}=0, r \neq q \\
\frac{\phi_{r}^{T} K^{I} \phi_{r}}{\phi_{r}^{T} M \phi_{r}}=\omega_{r}^{2}
\end{array}
$$

For synthetic data absent of measurement noise and fully consistent with the underlying analytical model, the matrix equality constraints in Equations 6, 9, and 10 can be applied in the constraint satisfaction problem to yield satisfiable solutions to the inverse problem. However, in practical applications, the matrix constraint equations need to be relaxed to account for measurement errors and the idealizations inherent within the discrete representation of the physical structure. In our treatment of this problem, measurement uncertainties are accounted for by relaxing the measured modal parameters from real numbers to intervals centered around the experimental estimates. In this relaxation, each partially described eigenvector, $\phi_{r}^{M}$, in the matrix containing the measured components of the mode shapes, $\Phi_{s \times m}^{M}$, is first scaled to unity maximum amplitude and then an interval is constructed around all but the maximum amplitude component of the measured mode shape as:

$$
\phi_{i, r}^{I, M}=\left[\underline{\phi}_{i, r}, \bar{\phi}_{i, r}\right]= \begin{cases}{\left[\phi_{i, r}^{M}-\chi_{i, r}, \phi_{i, r}^{M}+\chi_{i, r}\right]} & \text { if } \phi_{i, r}^{M} \neq 1 \\ {[1,1]} & \text { if } \phi_{i, r}^{M}=1\end{cases}
$$

where $\chi_{i, r}$ is a specified radius for the intervals. The motivation behind not relaxing the maximum amplitude component of the mode shape is to establish this component as a fixed reference for the eigenvector, since the amplitude of an eigenvector is not unique. Likewise, the experimentally measured eigenvalues can be prescribed as intervals:

$$
\omega_{r}^{I}=\left[\underline{\omega}_{r}, \bar{\omega}_{r}\right]
$$

to reflect uncertainty in the experimental estimates of the natural frequencies. These interval relaxations of the measured eigeninformation apply to the constraint equations in Equations 6 and 10.

The radius of the intervals can either be established based on a measure of uncertainty in the experimentally estimated modal parameters, if available, or can be iteratively assigned based on the solvability of the constraint satisfaction problem. If the radius is prescribed too low in the presence of measurement and idealization errors, the nonlinear constraint satisfaction processor will return no solutions indicating that there are no satisfiable solutions to the inverse eigenvalue problem. This would be analogous to establishing a constraint equation for the Cross-in-Tray function described in Section 2 with the function equated to a value below the global minimum. Fortunately, the IBEX nonlinear constraint satisfaction solver can very efficiently identify non-satisfiable cases, which allows for progressive widening of the radius, $\chi$, until satisfiable solutions are first yielded. In addition, one can continue to increase this radius to further relax the constraint equation, which can be used to provide a measure of how well conditioned the inverse problem is for the given uncertain parameters in the model as well as identify alternative solutions similar to those identified around the global minimum. This extension would be analogous to application of the relaxed 


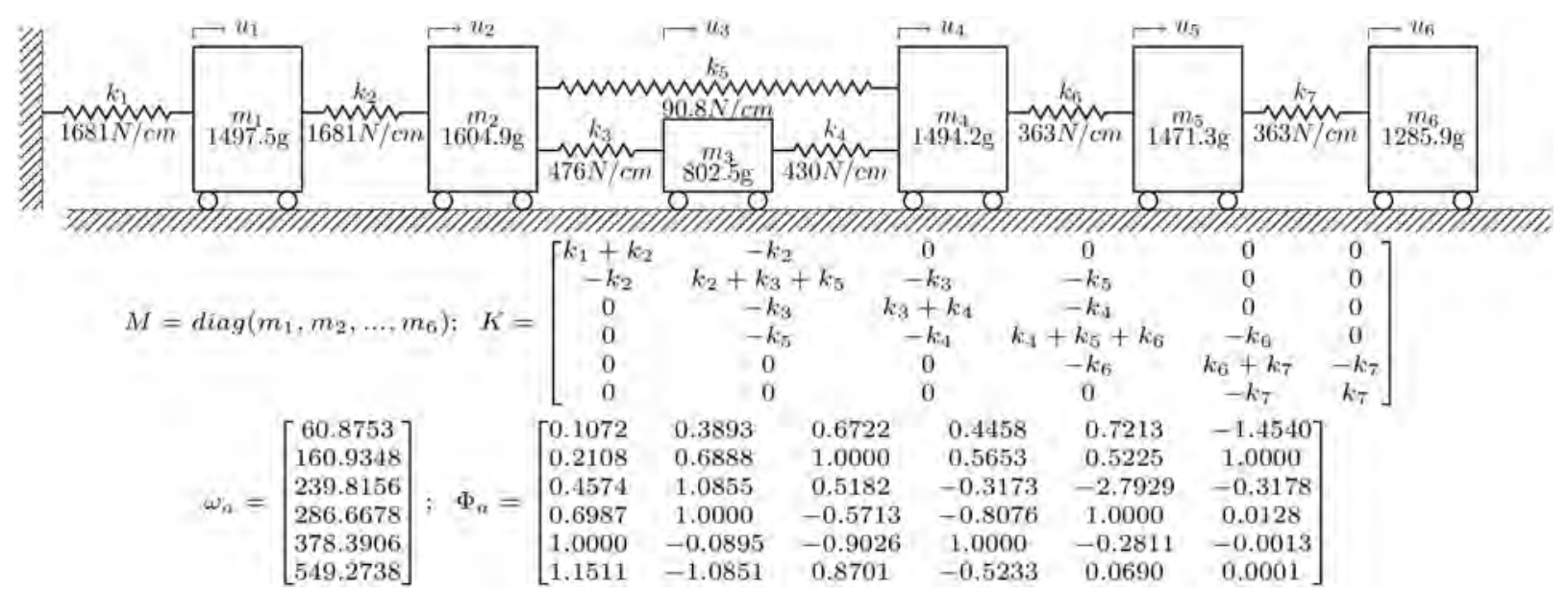

Figure 3: Six degree-of-freedom mass-spring system considered in numerical verification

inequality constraint for the Cross-in-Tray function described in Section 2, where the relative steepness of the basins around the global minimum and the presence of local minima were exposed. These unique features of the developed methodology are demonstrated for the model updating problem using both numerical and experimental data in the following sections of this paper.

\section{Numerical Verification of Methodology}

To demonstrate the capabilities of the formulated constraint satisfaction-based approach for the model updating problem, this study first numerically considers a 1st-order statically indeterminate spring-mass system with six degrees of freedom, shown in Figure 3. The structural configuration of this model was intentionally selected in order to generate ill-posed cases to the inverse eigenvalue problem admitting multiple solutions as well as spurious modes. Through linear parameterization of the model, elemental contributions to the stiffness matrix from each linear elastic spring are explicitly defined in the structured matrices. The mass and stiffness properties of the system were assigned to closely parallel an experimental analog to this numerical model that is subsequently presented in this paper for experimental validation. The eigenpairs of this undamped system were generated through eigendecomposition and are presented below the schematic representation of the MDOF model in Figure 3. The eigenvectors are normalized with respect to the measured sensors such that the magnitude of the largest entry in each eigenvector is one, rather than mass normalized. This is done to minimize any bias in the interval precision applied to the solution of the unmeasured components of each eigenvector when applying contractor programming to solve the constraint equations. Furthermore, the normalization serves to demonstrate that the generalized approach is applicable both to cases of model updating applied with results from experimental modal analysis (EMA), where mass normalization of the modes is possible, and operational modal analysis (OMA), where mass normalization of the modes is prohibited due to the lack of measurement of the input excitation forces.

\subsection{Application to a Well-Posed Problem}

To verify the framework on a well-posed problem, the seven spring stiffness assignments, $\left\{k_{1}, k_{2}, \ldots, k_{7}\right\}$ were treated as uncertain parameters in the model. In order to produce a partially described inverse eigenvalue problem with incompletely measured modes, synthetic measurements of the mode shapes were limited to those from degrees of freedom $u_{2}, u_{4}$, and $u_{5}$ and the eigeninformation was limited to the first three modes. Consequently, the eigenpairs 
provided to the finite element model updating problem were:

$$
\Omega=\left[\begin{array}{ccc}
60.8753 & 0 & 0 \\
0 & 160.9348 & 0 \\
0 & 0 & 239.8156
\end{array}\right] \quad \Phi=\left[\begin{array}{ccc}
\phi_{1,1} & \phi_{1,2} & \phi_{1,3} \\
0.2108 & 0.6888 & 1.0000 \\
\phi_{3,1} & \phi_{3,2} & \phi_{3,3} \\
0.6987 & 1.0000 & -0.5713 \\
1.0000 & -0.0895 & -0.9026 \\
\phi_{6,1} & \phi_{6,2} & \phi_{6,3}
\end{array}\right]
$$

where the measured eigenvector components are actually prescribed as intervals, $\left[\underline{\phi}_{i, r}, \bar{\phi}_{i, r}\right]$, centered around these values, as in Eq. 11. Since the unmeasured components of the mode shapes appear in the constraint equations formed from the generalized eigenvalue problem, the incomplete eigenvector matrix introduces nine additional unknowns to the seven unknown stiffness parameters. The search space for these 16 unknowns was constrained by introducing bounds on the uncertain parameters in the model, $k_{j} \in[0.1 \mathrm{~N} / \mathrm{cm}, 2500 \mathrm{~N} / \mathrm{cm}]$, and on the unmeasured components of the mode shapes, $\phi_{i, r} \in[-10,10]$. For this example, an interval precision of 25 for the uncertain stiffness assignments and 0.025 for the unmeasured components of the mode shapes were specified for the stopping criteria of the CSP. The difference in the specified required interval precisions was chosen to reflect the differences in the relative amplitude of the stiffness assignments compared to the components of the normalized mode shapes.

Despite the significant number of unknown variables in the coupled nonlinear constraint equations and the large search space associated with these unknowns, the nonlinear constraint solver was found to be capable of mapping the feasible solution space in only a few seconds of CPU time. Figure 4 presents the sets of interval vector solutions developed with three different radii of measurement uncertainty, $\chi$, introduced uniformly around the synthetic measurements of the three mode shapes. For the smallest radius, $\chi=0.0001$, the solution consists of three interval vectors, one of which contains the exact solution for the stiffness values and unmeasured components of the mode shapes. By increasing the radius associated with the measurement uncertainty, a set of interval vector solutions is developed that produce a basin around the exact solution. This ability to map the feasible solutions under a set of relaxed constraints provides a means of quantifying the uncertainty in the identified parameters of the model, while retaining the relationships between the parameters contained in the solution set. In this example, these basins reveal that the relative uncertainty associated with the inverse solution to parameters $k_{6}$ and $k_{7}$ with $\chi=0.001$ is very low (less than $\pm 0.4 \%$ ), while the conditioning of the inverse problem leads to larger uncertainty for parameters $k_{1}, k_{2}, k_{3}$, and $k_{4}$ (approximately $\pm 5 \%$ ) and the parameter with the greatest uncertainty is $k_{5}$ (approximately $\pm 10 \%$ ). It should also be noted here that refining the interval precision of the solutions was found to not significantly alter the basins of solutions yielded by the solver. However, by reducing the required interval precision at the expense of additional computational time, the boundary of each basin is refined to a smoother approximation as boxes with smaller width can more accurately map the curvature along this perimeter.

\subsection{Application to an Ill-Posed Problem}

Model updating has conventionally not been applied to problems that are ill-posed as a result of limited experimental data relative to the uncertain parameters in the model, since optimization schemes are not suitable for cases with non-unique solutions. However, framing the model updating problem as a CSP allows for solution of such problems and, as demonstrated in the following example, may introduce novel capabilities for parameter identification in structural health monitoring applications. Extension of this problem to an ill-posed case can be demonstrated by limiting the prescribed eigeninformation to the first two eigenvalues and the corresponding incomplete eigenvectors. In this case, the synthetic measurement data for the model updating problem is provided as:

$$
\Omega=\left[\begin{array}{cc}
60.8753 & 0 \\
0 & 160.9348
\end{array}\right] \quad \Phi=\left[\begin{array}{cc}
\phi_{1,1} & \phi_{1,2} \\
0.2108 & 0.6888 \\
\phi_{3,1} & \phi_{3,2} \\
0.6987 & 1.0000 \\
1.0000 & -0.0895 \\
\phi_{6,1} & \phi_{6,2}
\end{array}\right]
$$



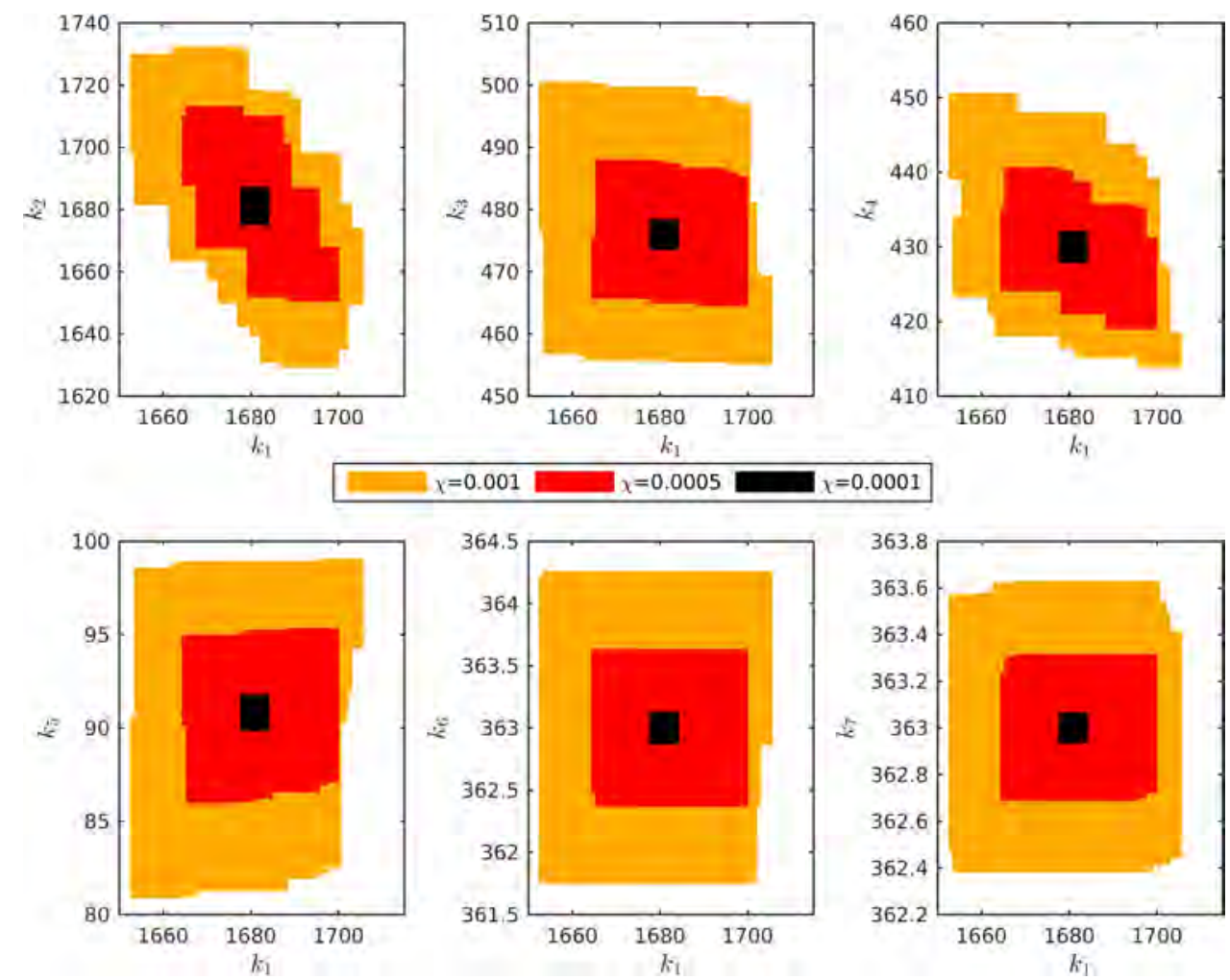

Figure 4: Feasible solutions for a well-posed analytical case of model updating with increasing uncertainty on the measured components of the mode shapes

Since the problem is ill-posed due to the non-uniqueness of the inverse solution, there are a large number of solutions to this inverse eigenvalue problem without having to introduce the interval relaxation on the measured components of the mode shapes. In developing the CSP for this problem, the same bounds previously enforced on the uncertain parameters and unmeasured components of the eigenvector matrix were specified. An interval precision of 2 for the uncertain stiffness parameters and 0.002 for the unmeasured components of the mode shapes were specified for the stopping criteria. Application of the IBEX nonlinear constraint solver for solution of the CSP using contractor programming produced 4,386 feasible interval vector solutions. Across all feasible solutions to the inverse eigenvalue problem, three distinct basins of interval solutions were discovered within the bounded search space. These basins of feasible solutions are presented for each of the seven stiffness assignments in Figure 5a, where the stiffness assignments in the model used to generate the synthetic data are presented as a marker in each subplot. It should be emphasized that, due to the intentionally ill-posed nature of the problem, all of the solutions obtained are correct solutions to the inverse eigenvalue problem subject to the partially described and incompletely measured eigeninformation matrices prescribed. Forward solution of the eigenvalue problem with the identified parameter sets was used to confirm that each of the feasible solutions correctly exhibits the two prescribed eigenvalues and corresponding measured components of the eigenvectors.

There are several important features of the set of feasible solutions developed that provide motivation for further exploration of this constraint satisfaction approach to model updating. First, a closer examination of the complete modal parameters produced by eigenvalue decomposition of the reconstructed models generated with each set of feasible parameter sets reveals that the solutions in two of the basins exhibit spurious modes. As indicated by Figure 5b, only the parameter sets contained within the first basin exhibit natural frequencies for the first two modes that match the prescribed eigenvalues. Parameter sets within the other two basins produce models where the natural frequencies of higher-order modes match the prescribed eigenvalues. Assuming that either vibration testing or engineering judgment is able to discredit the presence of any additional modes within the bandwidth of prescribed data, these basins 
of solutions could be discarded as physically implausible to reduce the feasible solution space.

Second, in addition to identifying the uncertain parameters in the model, the approach also identifies feasible values for the unmeasured components of the mode shapes in the prescribed eigenvector matrix. This permits the identification of spurious solutions, or feasible solutions that do not exhibit reconstructed mode shapes consistent with expectations, without requiring eigenvalue decomposition of the each feasible model, which can be computationally intensive for large analytical models. Figure 5c presents a comparison between the complete mode shapes from the original model and reconstructed mode shapes typical of each basin produced through constraint satisfaction. These comparisons reveal that the parameter sets admitting spurious modes can be readily identified by their reconstructed mode shapes, which differ significantly from the complete solution since they are higher-order modes. Therefore, with practitioner judgment is it possible to discard spurious solutions by simply evaluating the reconstructed mode shapes, which are included in the solution set and therefore can be evaluated with no additional computational expense in this methodology. This concept of integrating "human-in-the-loop" strategies for assessing alternative solutions within finite element model updating has been recently proposed by Caicedo and Yun [33] and is a possible extension of the current study that will be explored in future work. Additionally, techniques such as finding the natural, or minimumlength, solution with respect to the expected parameter values can be used to select updated parameter sets within under-determined problems [34].

Third, in addition to being able to describe the nonlinear interrelationships among spring stiffness assignments yielding feasible solutions, the basins establish the permissible range, or uncertainty, associated with each identified stiffness parameter. While the ill-posed nature of the problem explored produces a wide range of uncertainty across five of the spring stiffnesses, two spring stiffness assignments $\left(k_{6}\right.$ and $\left.k_{7}\right)$ were identified as constants within each basin. Consequently, the approach exactly identified uncertain parameters in a substructure of the model despite the severely limited data provided. It is particularly interesting to note that the nonlinear constraint solver exactly identifies the spring stiffness $k_{7}$ despite the absence of a measurement on the attached mass, $m_{6}$. The development of sensor placement strategies to produce parameter identification within targeted substructures may be an interesting future extension of this work.

\section{Experimental Application on a Shear Building Model}

To validate the application of the proposed methodology on an experimental dataset, a laboratory-scale six-story shear building structure was developed as an analog to the mass-spring model used in the numerical verification (Figure 6a). This structure was constructed of $15.2 \mathrm{~cm}$ deep and $1.3 \mathrm{~cm}$ thick aluminum plate masses serving as rigid diaphragm floors at each story elevation. The plate mass at the third floor elevation was $10.2 \mathrm{~cm}$ wide, while the other plate masses were $20.3 \mathrm{~cm}$ wide. These plates were supported by $15.2 \mathrm{~cm}$ wide aluminum sheets with different thicknesses, as indicated in Figure 6b, to vary their stiffness. An aluminum plate washer and row of four flange-head cap screws were used on each side of the plate masses to secure the sheets to each floor. Prior to experimental modal analysis, each cap screw was torqued to $1084 \mathrm{~N} \cdot \mathrm{cm}$ to ensure uniform connectivity. If the behavior of the test structure is idealized with the typical assumptions adopted for a shear building model, a six degree of freedom simplified analytical representation of the system can be constructed that corresponds to the model used for the numerical verification in the prior section, shown in Figure 3. A lumped mass matrix formulation was adopted for this study and the lumped masses used in the model were measured by weighing individual components of the structure. Likewise, estimations of the stiffness contributions for each of the aluminum sheets serving as columns were obtained by approximating each as a fixed-fixed beam subject to relative end deflection. Both the measured masses and approximated stiffness contributions in the model are presented in Figure 3 and were used in the prior numerical verification.

\subsection{Vibration Testing and System Identification}

Experimental modal analysis of the structure was conducted using six Measurement Specialties 4000A-002 singleaxis accelerometers featuring a full-scale range of $\pm 2 \mathrm{~g}, 0-200 \mathrm{~Hz}$ measurement bandwidth, and $35 \mu \mathrm{g} / \sqrt{\mathrm{Hz}}$ spectral noise. These vibration transducers were mounted to each plate mass of the test structure with a thin layer of wax to measure the in-plane lateral response in the weak direction of the building frame. A PCB Piezotronics 086C03 modally tuned impact hammer with super-soft tip was used to provide impulse excitation to the structure. A National Instruments PXIe-4497 24-bit simultaneously sampling dynamic signal analyzer was used for signal conditioning 

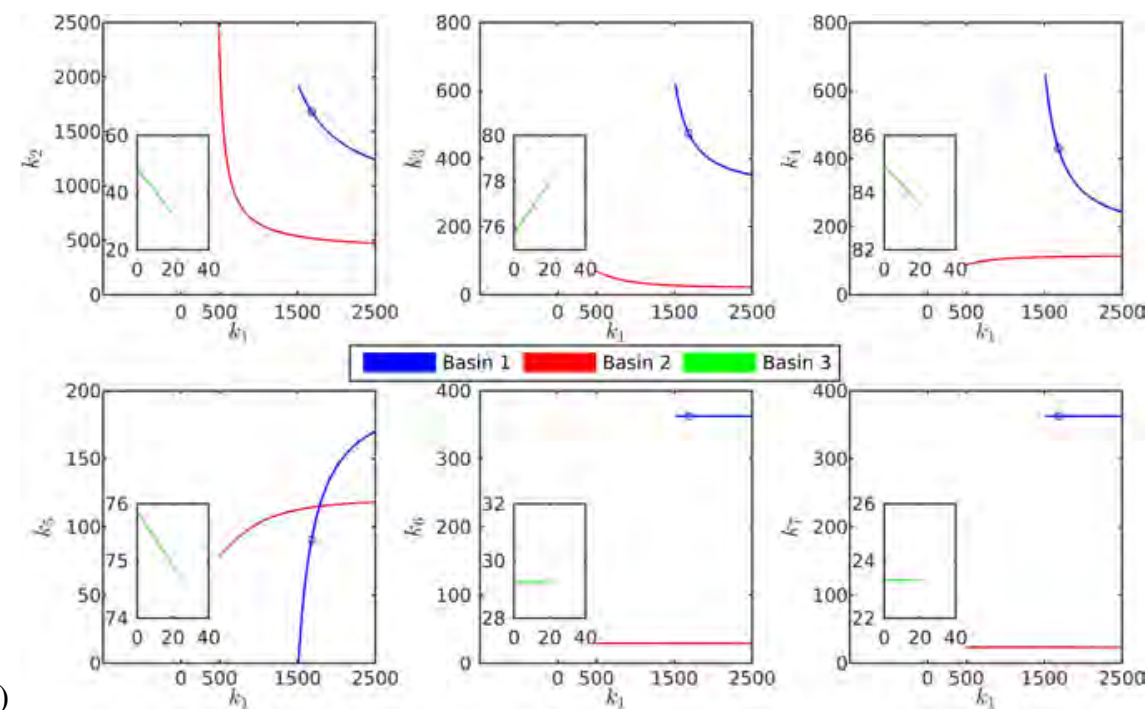

a.)

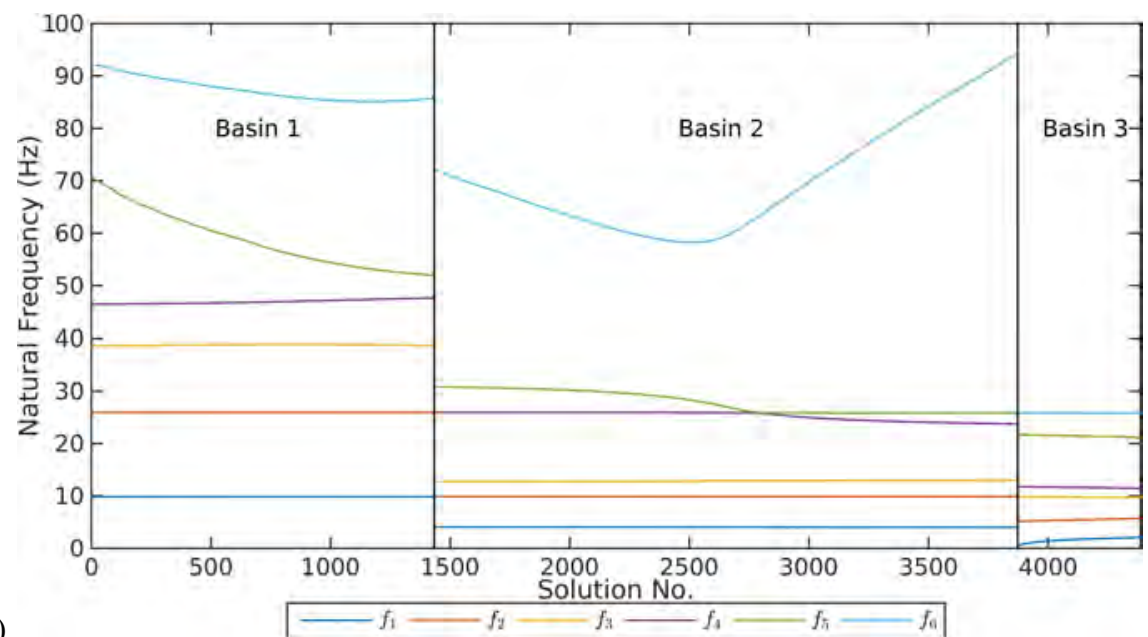

b.)
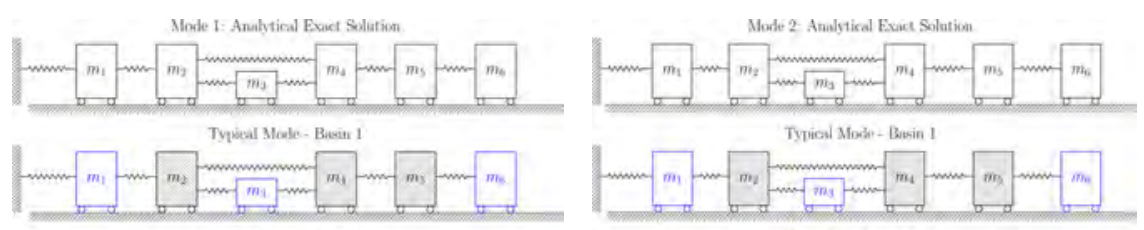

Typuas Moab - Busin 1

Typowal Mote- Basin 2
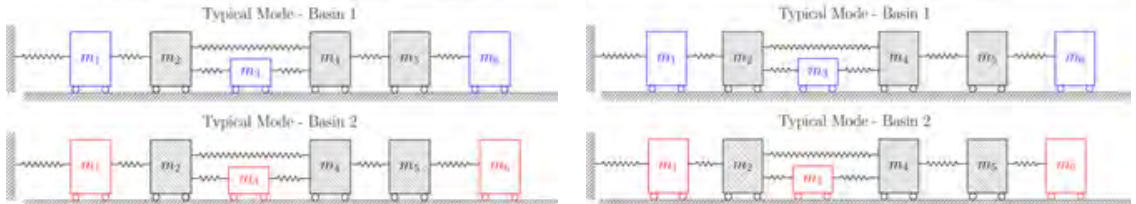

Typüical Mode - Busin 2

c.)
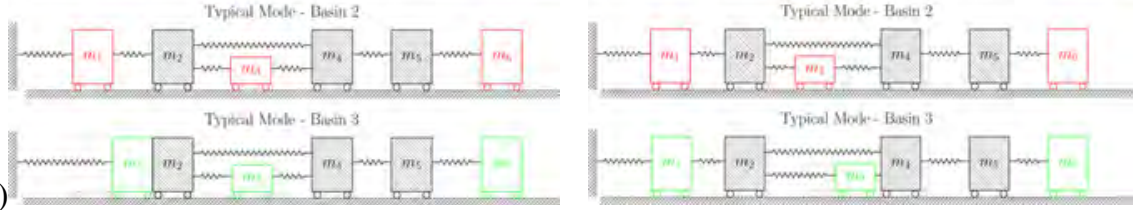

Figure 5: Complete set of feasible solutions for non-unique model updating problem with numerical data: a.) Basins of feasible stiffness assignments; b.) Natural frequencies associated with the feasible solutions; c.) Comparison of reconstructed mode shapes to the complete analytical modes from the reference structure 
and acquisition of the accelerometer signals as well as the impact force with a sampling rate of $10 \mathrm{kHz}$. Frequency response functions developed using the classical $H_{1}$ estimator [35] are presented in Figure $6 \mathrm{c}$ for the bandwidth encompassing the six modes associated with the response of shear building model in the weak-axis of bending.

Experimental estimates of the modal parameters were obtained through system identification using the combined deterministic stochastic subspace state-space system identification algorithm [36]. In application of this algorithm, experimental modal parameter estimates for the structure were calculated through eigendecomposition of the state matrix across a range of model orders and stabilization plots were utilized to select consistent pole estimates. In order to obtain improved estimates of modal parameters to attenuate effects of measurement and process noise, five stable poles and their corresponding eigenvectors were averaged for each experimental mode. The identified undamped natural frequencies are presented in Figure 7 along with the identified real-valued mode shapes.

\subsection{Experimental Application to a Well-Posed Problem}

Consistent with the numerical verification, the seven stiffness assignments were treated as uncertain parameters in the analytical model of the shear building structure and the experimental data was limited to measurements from degrees of freedom $u_{2}, u_{4}$, and $u_{5}$. To parallel the construction of the problem in Section 4.1, the partially-described and incomplete experimental eigeninformation was first provided to the nonlinear constraint solver as:

$$
\Omega=\left[\begin{array}{ccc}
61.2953 & 0 & 0 \\
0 & 165.8179 & 0 \\
0 & 0 & 245.3396
\end{array}\right] ; \quad \Phi=\left[\begin{array}{ccc}
\phi_{1,1} & \phi_{1,2} & \phi_{1,3} \\
0.2606 & 0.9582 & -0.9963 \\
\phi_{3,1} & \phi_{3,2} & \phi_{3,3} \\
0.7436 & 1.0000 & 1.0000 \\
1.0000 & -0.2527 & 0.7103 \\
\phi_{6,1} & \phi_{6,2} & \phi_{6,3}
\end{array}\right]
$$

with the measured eigenvector components are prescribed as intervals, $\left[\phi_{i, r}, \bar{\phi}_{i, r}\right]$, centered around the values shown with the uniform radius $\chi$. Likewise, the search space for unknowns in the constraint equations was established by bounding the uncertain parameters in the model to $k_{i} \in[0 \mathrm{~N} / \mathrm{cm}, 2500 \mathrm{~N} / \mathrm{cm}]$ and bounding the unmeasured components of the mode shapes to $\phi_{j, k} \in[-10,10]$. As in the numerical verification, an interval precision of 25 for the uncertain stiffness assignments and 0.025 for the unmeasured components of the mode shapes were specified for the stopping criteria of the CSP.

The nonlinear constraint solver was applied iteratively to the set of constraint equations by incrementing the size of the radius, $\chi$, around the measured eigenvector components until feasible solutions were generated. When the

a)

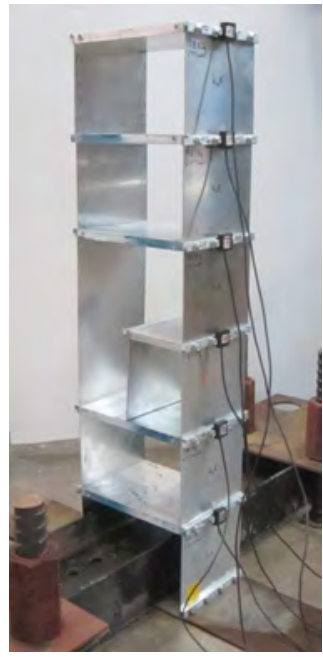

b)

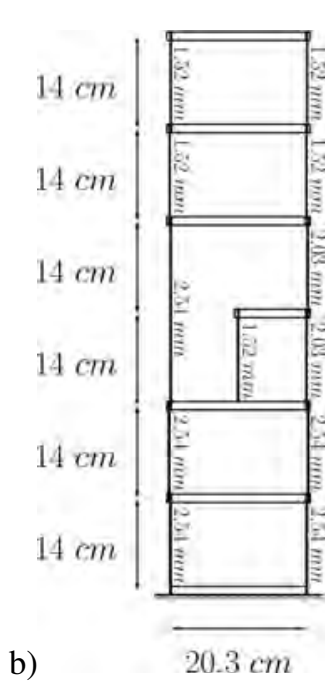

c)

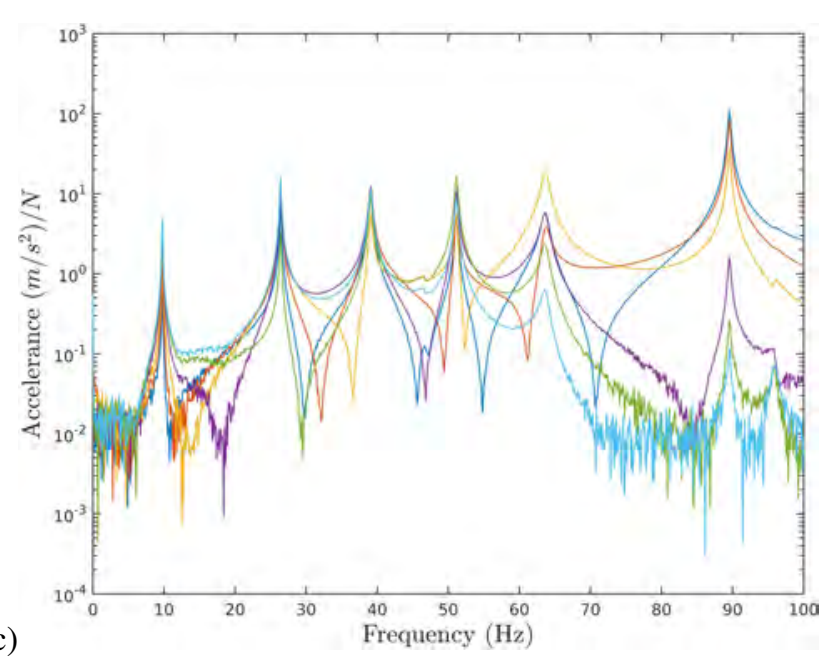

Figure 6: Laboratory six-story shear building model: a.) photograph of instrumented test structure, b.) structural geometry and thickness of aluminum sheet columns, c.) frequency response functions obtained from impact excitation at elevation of first story mass 


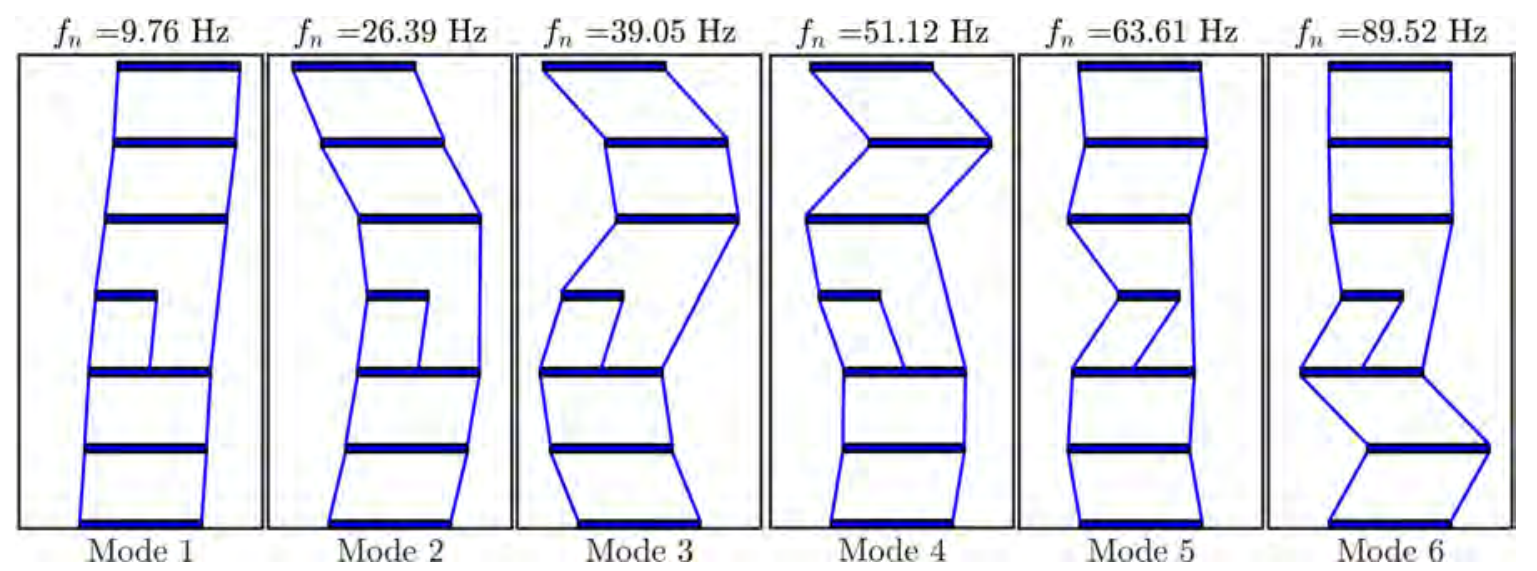

Figure 7: Undamped natural frequency and mode shape estimates of the test structure obtained through system identification

radius was increased to 0.0027 , the nonlinear constraint solver generated 3 solutions within the parameter search space in less than 10 seconds of computational time. To quantify the uncertainty in the parameter estimates and reflect the relative conditioning of the inverse problem for each uncertain parameter, the radius on the uncertain eigenvector measurements was further increased over two increments and the basins of solutions are presented in Figure 8. As in the numerical verification, these basins provide a means of assessing the relative uncertainties in individual parameters and indicate that relatively small changes in the measured components of the mode shapes result in admitting a large number of solutions for this problem. This is a reflection of the conditioning of this inverse problem, however the formulated approach allows for quantifying the sensitivity of the individual parameters to the measurement uncertainties. As in the numerical example, certain parameter estimates $\left(k_{6}\right.$ and $\left.k_{7}\right)$ are found to be less sensitive to measurement uncertainties and can be reliably identified in the presence of conditioning issues.

A representative interval solution for the uncertain parameters obtained from the original solution basin is presented in Table 1. Despite the large search space, the nonlinear constraint solver identified assignments for the stiffness parameters that are generally consistent with the idealized approximations. Discrepancies between the identified stiffness assignments and approximations can be partially attributed to the assumptions used to develop the theoretical estimates, material and fabrication tolerances in the construction of the experimental model, and idealization errors introduced by modeling this continuous system as a discrete MDOF system. Additionally, as demonstrated in Figure 8 , the problem is not particularly well-conditioned and, consequently, select parameter estimates are sensitive to measurement errors and could be improved by inclusion of additional eigeninformation in the inverse problem. To verify the interval solution, interval modal analysis was performed using the assumed mass matrix and the interval stiffness matrix constructed with the interval vector for the identified stiffness parameters. The interval eigenvalues for this system were then derived using the interval modal analysis technique presented in Sim et al. [37]. Table 2 provides a comparison between the experimentally estimated natural frequencies and the interval natural frequencies produced by the updated model. The solution is found to contain each of the three prescribed natural frequencies with a tight enclosure around the experimental estimates.

\subsection{Experimental Application to an Ill-Posed Problem}

Similar to the numerical verification, an ill-posed case with uniqueness challenges was also investigated using incomplete measurement data from the first two modes. In this example, the following partially-described and incompletely measured experimental eigeninformation matrices were provided as prescribed data for the model updating 

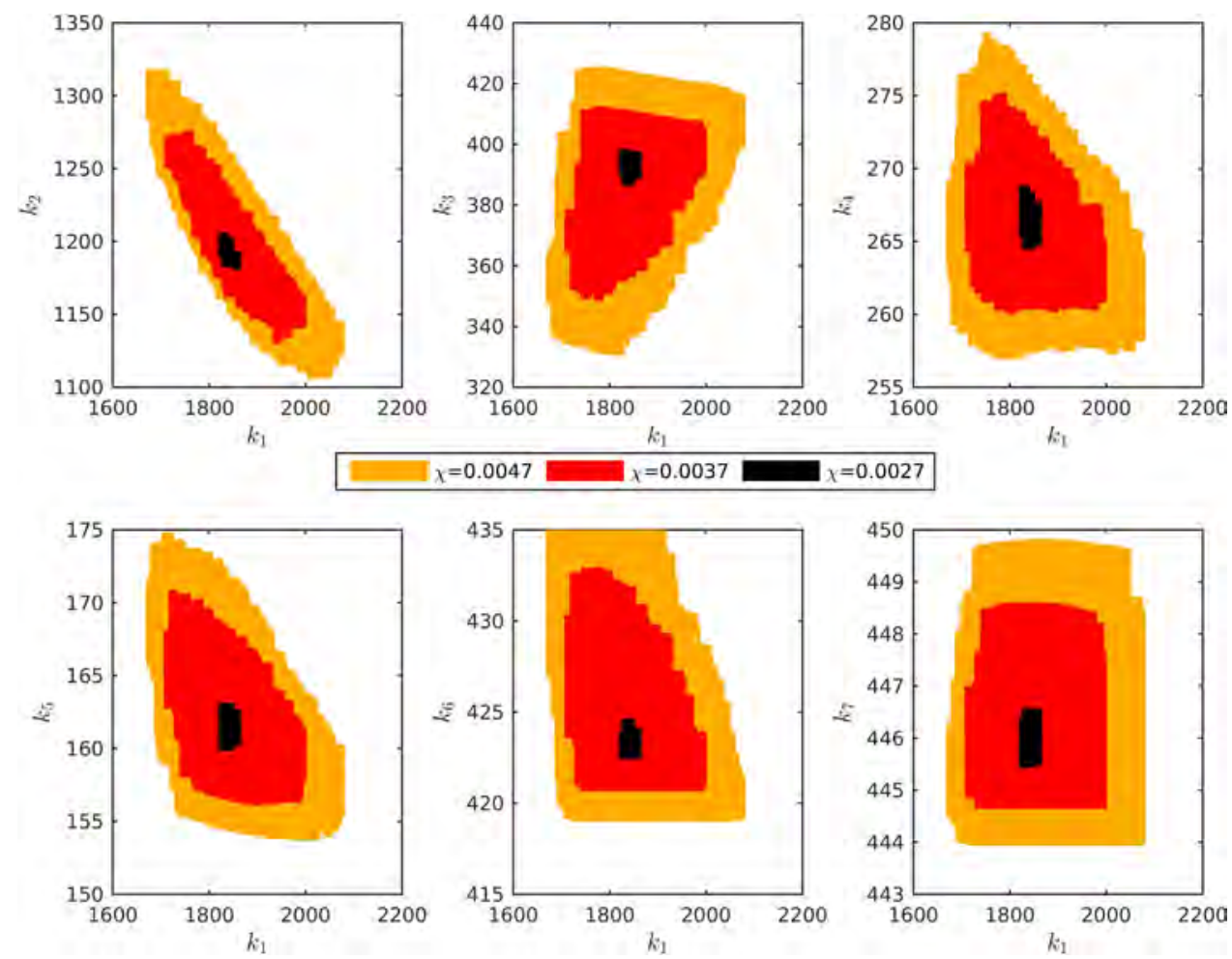

Figure 8: Feasible solutions for a well-posed experimental case of model updating with increasing uncertainty on the measured components of the mode shapes

problem:

$$
\Omega=\left[\begin{array}{cc}
61.2953 & 0 \\
0 & 165.8179
\end{array}\right] ; \quad \Phi=\left[\begin{array}{cc}
\phi_{1,1} & \phi_{1,2} \\
0.2606 & 0.9582 \\
\phi_{3,1} & \phi_{3,2} \\
0.7436 & 1.0000 \\
1.0000 & -0.2527 \\
\phi_{6,1} & \phi_{6,2}
\end{array}\right]
$$

The CSP for this problem was established with the same bounds on the unknown variables as the prior examples and, due to the ill-posed nature of the problem, the measured components of the mode shapes were not relaxed to interval estimates. An interval precision of 2 for the uncertain stiffness parameters and 0.002 for the unmeasured components of the mode shapes were specified for the stopping criteria. Application of the IBEX nonlinear constraint solver to the formulated constraint satisfaction problem yielded 5,890 feasible interval vector solutions with three distinct basins similar to those developed in the numerical verification discovered within the bounded search space (Figure 9a). Forward solution of the eigenvalue problem with the identified stiffness parameter sets was used to confirm that each of the feasible solutions correctly exhibit the two prescribed eigenvalues and corresponding measured components of the eigenvectors within the prescribed relaxation tolerance. As in the numerical example, two of the basins of feasible solutions were found to exhibit spurious mode development. For these combinations of stiffness parameter assignments, the updated model gives rise to additional eigenvalues within the frequency bandwidth of the two prescribed eigenvalues (Figure 9b). Typical reconstructed mode shapes for each basin using the identified missing components of the prescribed mode shapes are provided in Figure 5c, which parallel the solutions obtained with the numerical example to further validate the extension of the proposed methodology to experimental data. In addition, two of the uncertain parameters in the model $\left(k_{6}\right.$ and $\left.k_{7}\right)$ were exactly identified in all three of the solution basins, despite the ill-posed nature of the problem. Furthermore, the identified assignments for these parameters in the first basin agree with those identified in the previous solution to the well-posed problem. 

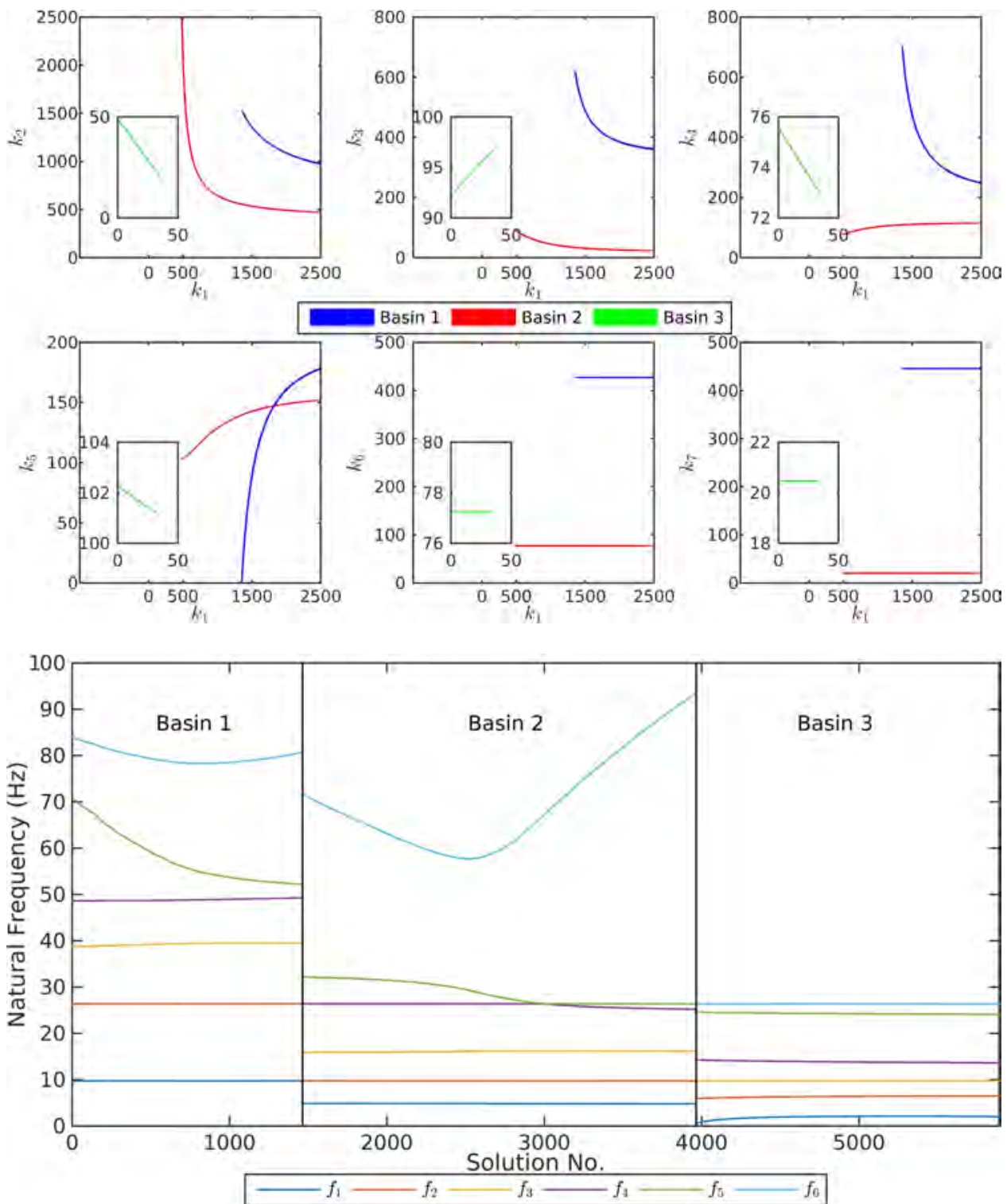

b.)
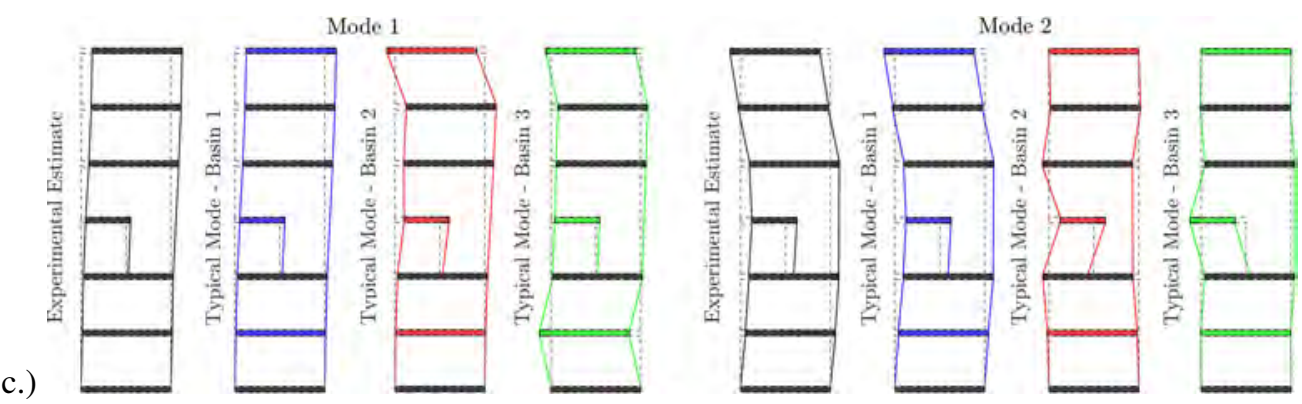

Figure 9: Complete set of feasible solutions for non-unique model updating problem with experimental data: a.) Basins of feasible stiffness assignments; b.) Natural frequencies associated with the feasible solutions; c.) Comparison of reconstructed mode shapes to the complete experimental measurements 
Table 1: Comparison of stiffness estimates to experimental results obtained using three incompletely measured eigenpairs

\begin{tabular}{|c|c|}
\hline Parameter & Interval Solution, $\left[\underline{k}_{j}, \bar{k}_{j}\right]$ \\
\hline \hline$k_{1}$ & {$[1850.4,1864.6]$} \\
\hline$k_{2}$ & {$[1181.0,1192.8]$} \\
\hline$k_{3}$ & {$[388.9,397.4]$} \\
\hline$k_{4}$ & {$[264.7,267.7]$} \\
\hline$k_{5}$ & {$[160.2,162.6]$} \\
\hline$k_{6}$ & {$[422.5,424.1]$} \\
\hline$k_{7}$ & {$[445.5,446.6]$} \\
\hline
\end{tabular}

Table 2: Comparison between experimental modal estimates and updated model

\begin{tabular}{|c|c|c|c|}
\hline Mode & $f_{\exp }$ & {$[\underline{f}, \bar{f}]$} & {$[\Delta \underline{f}(\%), \Delta \bar{f}(\%)]$} \\
\hline \hline 1 & 9.7555 & {$[9.7251,9.7786]$} & {$[-0.3110,0.2377]$} \\
\hline 2 & 26.3907 & {$[26.3343,26.4080]$} & {$[-0.2137,0.0653]$} \\
\hline 3 & 39.0470 & {$[39.0123,39.0550]$} & {$[-0.0888,0.0204]$} \\
\hline
\end{tabular}

\section{Comparison to Bayesian Model Updating Approaches}

As noted in the introduction, the developed methodology offers an alternative to probabilistic methods of finite element model updating. Beyond simply accounting for the challenges of measurement uncertainty in model updating, nonlinear constraint satisfaction provides unique advantages and capabilities that are not offered by current probabilistic techniques. To highlight these differences, particularly with respect to addressing the effects of measurement uncertainty on the parameter estimation, the challenges and limitations of probabilistic model updating are briefly reviewed and a comparison between probabilistic model updating and the nonlinear constraint satisfaction approach is presented using a simple benchmark example problem.

In contrast to the constraint satisfaction methodology presented in this paper, Bayesian model updating approaches rely on an likelihood function to quantify the model correlation. When modal data is used, the likelihood function includes a modal measure of fit function that incorporates an objective function for the natural frequencies and an objective function for the partially described mode shapes. For illustration, a commonly used modal measure of fit, provided by [38], is:

$$
J(\theta)=\sum_{r=1}^{N_{m}} \sum_{j=1}^{N_{s}}\left[\left(\frac{\left(1-\hat{\omega}_{r, j}^{2} / \omega_{r}^{2}\right)^{2}}{\varepsilon^{2}}\right)+\left(\frac{\phi_{r}^{T}\left(I-\hat{\phi}_{r, j} \hat{\phi}_{r, j}^{T}\right) \phi_{r}}{\delta^{2}\left\|\phi_{r}\right\|^{2}}\right)\right]
$$

where $N_{m}$ is the number of modes included in the identification, $N_{s}$ is the number of sets of modal data, $\hat{\omega}_{r, j}$ and $\hat{\phi}_{r, j}$ are the experimental natural frequency and incomplete mode shape, respectively, for the $r$-th mode of the $j$-th data set, $\omega_{r}$ and $\phi_{r}$ are the corresponding natural frequency and incomplete mode shape for the model with parameter assignments $\theta$, and $\varepsilon$ and $\delta$ are parameters that reflect the uncertainty in the experimentally measured natural frequencies and mode shapes, respectively. There are several noteworthy issues associated with the use of the modal measure of fit in Bayesian model updating. First, the updating results can be very sensitive to the weighting coefficients, $\varepsilon^{2}$ and $\delta^{2}$, that express the relative importance of the modal frequency and partially described mode shape correlations to the objective function [39]. Second, direct matching of modes is required, which can be challenging with incompletely measured mode shapes [40]. Lastly, the measure of fit aggregates the model correlations and therefore does not ensure that each of the individual modal parameters of the identified modal are consistent with the experimental measurements within prescribed uncertainty bounds.

Additional challenges associated with Bayesian model updating stem from the need to evaluate multidimensional integrals that can not be solved analytically. A favored approach, particularly for models with many parameters, 
has been to leverage stochastic simulation methods, such as Metropolis-Hastings or Gibbs sampling, to develop the posterior probability density functions for the uncertain parameters. However, such approaches introduce several additional challenges. First, since the parameter space is evaluated by discrete sampling, the development of posterior joint probability distribution functions can be computationally intensive, particularly if either the size of the model or the uncertainty in the prior distributions is large. Second, the specification of the prior distributions influences the development and accuracy of the posterior probability distribution functions [41]. Transitional, or adaptive, methods have been introduced to address this later challenge by converging on the posterior distribution through intermediate probability distribution functions [42]. However, these methods introduce additional complexity and computational cost. Lastly, stochastic simulation methods are sensitive to user specified settings, such as the number of iterations before adaptation of the intermediate probability distribution functions, and can not guarantee complete exploration of the parameter search space and complete mapping of feasible solutions.

A two degree of freedom mass spring model sourced from [39] is used to illustrate these limitations within Bayesian model updating approaches and further demonstrate advantages of the proposed methodology. Consistent with the case presented in the literature, the structural matrices for this model are taken as:

$$
M=\left[\begin{array}{ll}
1 & 0 \\
0 & 1
\end{array}\right] \quad, \quad K=2000\left[\begin{array}{cc}
\theta_{1}+\theta_{2} & -\theta_{2} \\
-\theta_{2} & \theta_{2}
\end{array}\right]
$$

where $\theta_{1}$ and $\theta_{2}$ are uncertain stiffness parameters. Synthetic measurement data was generated for 15 sets of modal parameter estimates by generating Gaussian distributions with coefficient of variation of 0.01 around the two natural frequencies and mode shapes for the model specified with $\theta_{1}=\theta_{2}=1$. For Bayesian model updating, an adaptive Metropolis-Hastings Markov Chain Monte Carlo method was used to sample posterior probability distribution functions with a likelihood function incorporating the modal measure of fit function in Equation 13. For all cases described, a uniform prior distribution was assigned to the two uncertain parameters in the model over the range [0,3]. Relative weighting of the natural frequency and mode shape terms in the modal measure of fit function was adjusted to yield three representative cases. The first two cases serve to illustrate the sensitivity of the Bayesian model updating results to the assignment of the relative weighting coefficients prescribed to the mode shape and natural frequency correlations in the modal measure of fit function. When the relative weighting is prescribed in a way that over-expresses the mode shape correlations relative to the natural frequency correlations, an under-determined problem is developed and the posterior distribution of the Markov Chain samples suggest non-uniqueness of the uncertain parameters (Figure 10a). When the relative weighting is prescibed in a way that over-expresses the natural frequency correlations relative to the mode shape correlations, the posterior distribution of the Markov Chain samples suggest two alternative solutions (Figure 10b). Only when the relative weighting is prescribed such that the natural frequency and mode shape correlations are balanced to express an appropriate degree of importance of each to the modal measure of fit is a posterior distribution uniquely generated around the two stiffness parameters used to develop the measurement data (10c). It should be noted that strategies for determining balanced weighting factors for Bayesian model updating have been developed [39], however these strategies require an additional layer of heuristic model updating on the relative weighting assignments to assess and select the appropriate model class. Consequently, these approaches further increase the computational burden associated with probabilistic model updating.

Constraint equations for the same two degree of freedom mass spring model were developed using the new methodology proposed by the authors. The standard deviations, $\sigma$, of the synthetic measurements of the natural frequencies and mode shapes were used to establish interval assignments about the mean of these modal parameters within the constraint equations. Nonlinear constraint satisfaction using interval arithmetic and contractor programming was then used to map all feasible solutions within a search space developed by bounding the parameters in the model using $\theta_{j} \in[0,3]$. In order to generate solutions for non-unique cases analogous to those presented for Bayesian model updating, the synthetic measurement data provided in the constraint equations was first restricted to either the mode shapes (Figure 11a) or the natural frequencies (Figure 11b). In both cases, subpavings were generated for interval uncertainties on the prescribed measurement components using confidence intervals of $\pm 2 \sigma, \pm \sigma$, and $\pm \frac{1}{2} \sigma$. In both cases, the nonlinear constraint satisfaction approach correctly identifies and maps the non-unique solutions in basins consistent with the Bayesian posterior distributions. However, unlike the samples of the posterior distributions produced by Bayesian model updating that require further statistical inference to estimate the probabilities associated with the parameter assignments, the nonlinear constraint satisfaction approach successfully propagates the measurement uncertainty through the inverse problem to completely and crisply map the associated confidence 

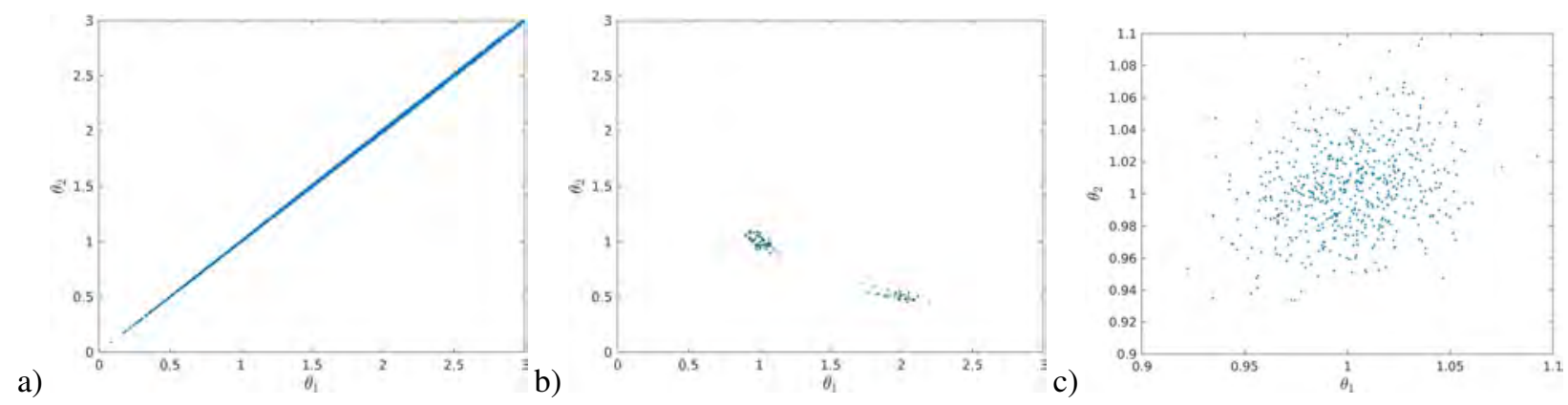

Figure 10: Samples of the posterior distribution obtained from Bayesian model updating of two degree of freedom model with uncertain stiffness parameters: a) mode shapes over-expressed in the measure of fit; b) natural frequencies over-expressed in the measure of fit; c) balanced model class

a)

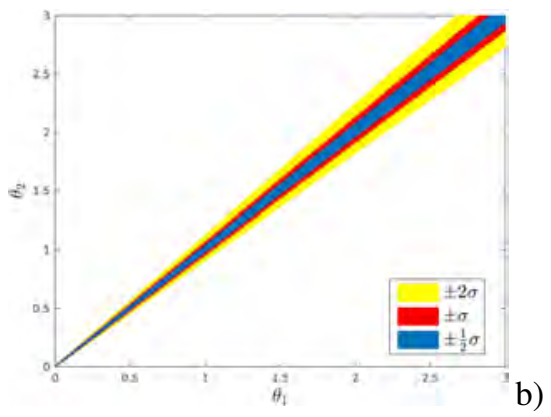

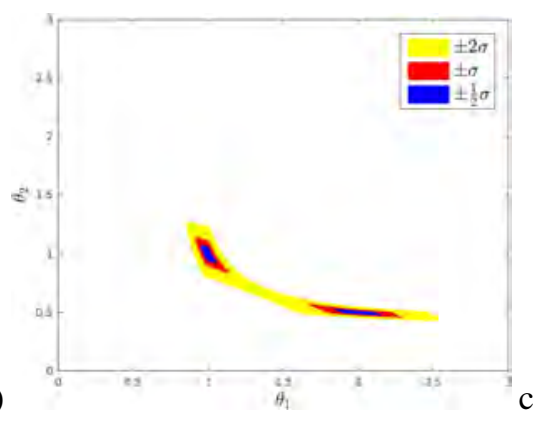

c)

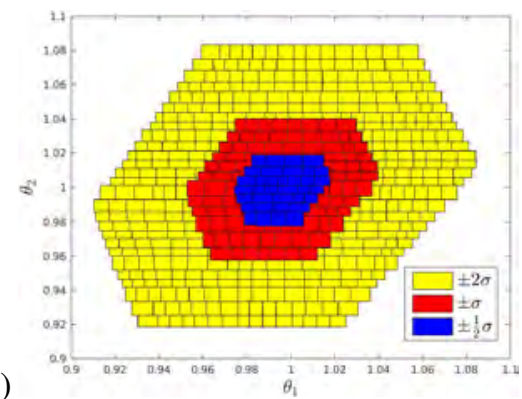

Figure 11: Subpavings of feasible solution space for parameters in two degree of freedom model: a) using only the mode shape measurements in the constraint equations; b) using only the natural frequency measurements in the constraint equations; c) using both the mode shape and natural frequency measurements

in the identified feasible parameter assignments. Furthermore, it should be emphasized that these non-unique cases need to be intentionally generated by omitting measurement data in the constraint equations, whereas they can arise unintentionally within the Bayesian method if relative weighting coefficients are prescribed in an unbalanced manner. When the synthetic data prescribed in the constraint equations includes both the natural frequencies and mode shapes, the nonlinear constraint solver correctly identifies the stiffness parameters used to generate the modal parameter data and encloses the confidence in the parameter estimates through the subpavings of the search space (Figure 11c). Comparison with current probabilistic model updating approaches, illustrates the advantages of both being able to directly specify the measurement uncertainty and completely enclose the feasible parameter space associated with the uncertain measurements using the nonlinear constraint satisfaction approach. In the Bayesian approach, the modal measure of fit aggregates all of the model correlations, which can not guarantee that modal parameters of the identified models strictly adhere to prescribed uncertainty bounds for individual parameters. Furthermore, statistical inference of the posterior samples is required to estimate parameter confidence, as opposed to the new methodology that crisply encloses the confidence intervals.

In terms of computational efficiency, the nonlinear constraint solver was able to map the feasible solution space to the nonlinear constraint satisfaction problem in a fraction of a second, while the Metropolis-Hastings Markov Chain Monte Carlo method used to perform Bayesian Model updating required several seconds to converge on the posterior distribution presented. It should be noted again that the computational time associated with the Bayesian method does not account for additional time required to select appropriate relative weighting factors. Since the nonlinear constraint satisfaction approach operates directly on the governing mechanics equations rather than objective functions that aggregate model correlations, potentially time consuming selection of model class is not required within the proposed methodology. 


\section{Conclusion}

A novel technique for structural identification through model updating has been presented by formulating the inverse problem as a constraint satisfaction problem. The formulation structures a set of coupled, nonlinear constraint equations over a bounded search space and efficiently obtains a complete set of feasible solutions to the constraint equations using interval arithmetic and contractor programming. The use of interval arithmetic in the approach has been further leveraged to introduce relaxation of the constraint equations to accommodate uncertainties in the model and measurements. Through numerical verification as well as application to an experimental dataset, unique capabilities of this methodology for the model updating problem have been demonstrated.

Foremost, the approach is capable of completely mapping feasible solutions to the structured inverse eigenvalue problem, which offers significant advantages over optimization and stochastic sampling techniques. An efficient process of progressive relaxation of the constraints equations to accommodate measurement uncertainties is used to identify the global optimal solution. Through further relaxation, the sensitivity of the parameter estimates to the measurement data can be revealed to quantify uncertainty in the parameter estimates associated with the conditioning of the problem as well as expose alternative solutions residing in local minima. Consequently, the approach provides a means of projecting uncertainty in the measurements to the parameter space and can permit future research to explore the incorporation of "human-in-the-loop" practitioner heuristics for the evaluation of alternative solutions to the global minimum. Furthermore, an additional unique capability of the approach is the demonstrated ability of the solver to completely enumerate the feasible solutions to ill-posed formulations of the model updating problem. Despite the uniqueness issues resulting from the limited measurement data used in the ill-posed form, results from the examples presented in this paper reveal the capability of this approach to exactly identify select uncertain parameters in substructures of the model. This capability may provide the foundation for developing novel vibration-based structural health monitoring strategies for condition assessment of local substructures with limited sensor deployments.

Future work will be directed toward leveraging the capabilities offered by this approach to challenges in vibrationbased damage detection. In addition, the exploration of specific contractors and design of contraction strategies beyond the default solver of the IBEX library offer the potential to enhance the performance of the methodology. Furthermore, the incorporation of model reduction strategies into the constraint equations may provide a means of improving the efficiency of the algorithm by eliminating the need to solve for unmeasured components of the eigenvectors. Lastly, the scalability of the proposed technique to larger structural models with more degrees of freedom will need to be investigated to extend the application to structures of greater complexity.

\section{Acknowledgment}

This material is based upon work supported by the National Science Foundation under Grant No. 1331825. Any opinions, findings, conclusions, or recommendations expressed in the material are those of the authors and do not necessarily reflect the views of the sponsor.

\section{References}

[1] A. Aktan, J. Brownjohn, Structural identification: opportunities and challenges, Journal of Structural Engineering 139 (2013) $1639-1647$.

[2] M. Chu, G. Golub, Inverse Eigenvalue Problems: Theory, Algorithms, and Applications, Oxford University Press, Oxford, UK, 2005.

[3] P. Avitabile, J. O’Callahan, Frequency response function expansion for unmeasured translation and rotation dofs for impedance modelling applications, Mechanical systems and signal processing 17 (2003) 723-745.

[4] J. E. Mottershead, M. Link, M. I. Friswell, The sensitivity method in finite element model updating: a tutorial, Mechanical systems and signal processing 25 (2011) 2275-2296.

[5] P. G. Bakir, E. Reynders, G. De Roeck, An improved finite element model updating method by the global optimization technique coupled local minimizers, Computers \& Structures 86 (2008) 1339-1352.

[6] L. Deng, C. Cai, Bridge model updating using response surface method and genetic algorithm, Journal of Bridge Engineering 15 (2009) 553-564.

[7] T. Marwala, Finite-element-model updating using computional intelligence techniques, Springer, 2010.

[8] A. Morassi, S. Tonon, Dynamic testing for structural identification of a bridge, Journal of Bridge Engineering 13 (2008) $573-585$.

[9] F. N. Çatbaş, T. Kijewski-Correa, A. E. Aktan, Structural identification of constructed systems, Reston (VI): American Society of Civil Engineers (2013). 
[10] M. I. Friswell, J. E. Mottershead, H. Ahmadian, Finite-element model updating using experimental test data: parametrization and regularization, Philosophical Transactions of the Royal Society of London A: Mathematical, Physical and Engineering Sciences 359 (2001) $169-186$.

[11] J. L. Beck, L. S. Katafygiotis, Updating models and their uncertainties. I: Bayesian statistical framework, Journal of Engineering Mechanics 124 (1998) 455-461.

[12] J. Carvalho, B. N. Datta, A. Gupta, M. Lagadapati, A direct method for model updating with incomplete measured data and without spurious modes, Mechanical Systems and Signal Processing 21 (2007) 2715-2731.

[13] Y.-C. Kuo, B. N. Datta, Quadratic model updating with no spill-over and incomplete measured data: Existence and computation of solution, Linear Algebra and its Applications 436 (2012) 2480-2493.

[14] H. U. Köylüoglu, A. S. Çakmak, S. R. Nielsen, Interval algebra to deal with pattern loading and structural uncertainties, Journal of engineering mechanics 121 (1995) 1149-1157.

[15] D. Moens, D. Vandepitte, A survey of non-probabilistic uncertainty treatment in finite element analysis, Computer methods in applied mechanics and engineering 194 (2005) 1527-1555.

[16] R. Madarshahian, J. M. Caicedo, B. A. Zárate, Using P-Box and PiFE to Express Uncertainty in Model Updating, in: Topics in Model Validation and Uncertainty Quantification, Volume 5, Springer, 2013, pp. 81-88.

[17] S. Gabriele, C. Valante, An interval-based technique for FE model updating, Int. J. Reliability and Safety 3 (2009) 79-103.

[18] S. Gabriele, The interval intersection method for fe model updating, in: Journal of Physics: Conference Series, volume 305, IOP Publishing, 2011, p. 012091.

[19] H. Khodaparast, J. Mottershead, K. Badcock, Interval model updating with irreducible uncertainty using the Kriging predictor, Mechanical Systems and Signal Processing 25 (2011) 1204-1226.

[20] S.-E. Fang, Q.-H. Zhang, W.-X. Ren, An interval model updating strategy using interval response surface models, Mechanical Systems and Signal Processing 60 (2015) 909-927.

[21] C. Mares, J. Mottershead, M. Friswell, Stochastic model updating: Part 1- theory and simulated example, Mechanical Systems and Signal Processing 20 (2006) 1674-1695.

[22] R. Dechter, Constraint Processing, Morgan Kaufmann, 2003.

[23] R. Rossi, P. van Beek, T. Walsh, Handbook of Constraint Programming (Foundations of Artificial Intelligence), Elsevier Science, 2006.

[24] P. Hentenryck, The OPL Optimization Programming Language, MIT Press, 1999.

[25] E. Hyvnen, Constraint reasoning based on interval arithmetic: the tolerance propagation approach, Artificial Intelligence 58 (1992) $71-112$.

[26] G. Alefeld, D. Claudio, The basic properties of interval arithmetic, its software realizations and some applications, Computers \& Structures 67 (1998) 3-8.

[27] G. Chabert, L. Jaulin, Contractor programming, Artificial Intelligence 173 (2009) 1079-1100.

[28] L. Jaulin, Applied interval analysis: with examples in parameter and state estimation, robust control and robotics, volume 1, Springer Science \& Business Media, 2001.

[29] G. Chabert, IBEX C++ library for constraint processing over real numbers, 1999. URL: http://www.ibex-lib.org.

[30] MATLAB, version 8.6.0 (R2015b), The MathWorks Inc., Natick, Massachusetts, 2015.

[31] F. Benhamou, F. Goualard, L. Granvilliers, J.-F. Puget, Revising hull and box consistency, in: Logic Programming: Proceedings of the 1999 International Conference on Logic Programming, MIT press, 1999, p. 230.

[32] B. Neveu, G. Trombettoni, I. Araya, Adaptive constructive interval disjunction: algorithms and experiments, Constraints 20 (2015) $452-467$.

[33] J. M. Caicedo, G. Yun, A novel evolutionary algorithm for identifying multiple alternative solutions in model updating, Structural Health Monitoring 10 (2011) 491-501.

[34] S. J. Hu, H. Li, A systematic linear space approach to solving partially described inverse eigenvalue problems, Inverse Problems 24 (2008) $1-12$.

[35] A. Brandt, Noise and Vibration Analysis: Signal Analysis and Experimental Procedures, John Wiley \& Sons, West Sussex, UK, 2011.

[36] P. Van Overschee, B. De Moor, Subspace Identification for Linear Systems: Theory - Implementation - Applications, Kluwer Academic Publishers, 1996.

[37] J. Sim, Q. Zhiping, W. Xiaojun, Modal analysis of structures with uncertain-but-bounded parameters via interval analysis, Journal of Sound and Vibration 303 (2007) 29-45.

[38] M. Vanik, J. Beck, S. Au, Bayesian Probabilistic Approach to Structural Health Monitoring, Journal of Engineering Mechanics 126 (2000) $738-745$.

[39] B. Goller, J. Beck, G. Schuëller, Evidence-Based Identification of Weighting Factors in Bayesian Model Updating Using Modal Data, Journal of Engineering Mechanics 138 (2012) 430-440.

[40] H. Sun, O. Büyüköztürk, Probabilistic updating of building models using incomplete modal data, Mechanical Systems and Signal Processing 75 (2016) 27-40.

[41] J. Beck, S. Au, Bayesian Updating of Structural Models and Reliability Using Markov Chain Monte Carlo Simulation, Journal of Engineering Mechanics 128 (2002) 380-391.

[42] J. Ching, Y.-C. Chen, Transitional Markov Chain Monte Carlo Method for Bayesian Model Updating, Model Class Selection and Model Averaging, Journal of Engineering Mechanics 133 (2007) 816-832. 